\title{
An architectural approach to process control of gas compressor stations with a low temperature separation gas preparation unit based on a digital twin
}

\author{
Yu.V. Kozhukhov ${ }^{1}$, R. S. Marchenko ${ }^{1}$, I.V. Ilyin $^{1}$, A.A. Aksenov ${ }^{1 *}$ and Minh Hai Nguyen ${ }^{2}$ \\ ${ }^{1}$ Peter the Great St. Petersburg Polytechnic University Federal State Autonomous Educational Institution of Higher Education, St. \\ Petersburg, Russia. \\ ${ }^{2}$ Petrovietnam Exploration Production Corporation, Ho Chi Minh City, Viet Nam
}

\begin{abstract}
This article lists a part of a study of possibilities of using and integrating digital twins of the existing high-tech enterprise processes on the examples of a high-pressure gas compressor station and a gas treatment plant using low-temperature separation of an oil and gas enterprise using an architectural approach. The need for the use of digital twins (DT) in the high-tech industry is due to the inability to effectively conduct full-scale tests to select the necessary operating modes in the implementation framework of technological processes with varying parameters (high cost and increased resource consumption compared to the creation of a DT). The main difference between DT and conventional models is the threedimensional matrix of input variable parameters that can be given to DT for processing in order to obtain the most correct output data when performing simulations. At the same time, millions of operational cycles can be generated in real time, which allows us to most effectively solve various business problems. For example, the task of finding optimal solutions and prototype configurations under study.
\end{abstract}

\section{Introduction}

Today discontinuous market sets before all high-tech companies, the modernization of complex problems and improvement business management systems. The economic of digital transformation is setting new rules for businesses, in order to keep up with competitors and continue to grow. Digital economy allows the company to provide more flexible and competitive production and be as efficient as possible. When we talk about the digital economy, we mean data volume exponential growth rate of acceleration. Data is generated by companies and people. For clarity, the company "Domo" research results based on its analytical platform can be mentioned. Studies show that $90 \%$ of existing data appeared only during the last two years in the world.

Today, data is a company asset. They help to competently build and automate production and technological processes. Effective data collection and processing is a question. Required to resort to the data architecture construction.

Data architecture allows you to organize the process of collecting and processing information. So that the processed and cleaned data are correctly integrated with existing enterprise IT systems of enterprises and are an effective basis for making production and management decisions. To realize all the aims in the complex, it is advisable to apply an architectural approach.

In modern enterprises, there is an increasing need to implement the so-called "digital twin". Digital twin is a highly detailed digitized object (process) or complex object (processes) model.

Their application is not limited only to objects and their characteristics - production entire technological processes digital twins are currently being created, due to the need to select the optimal parameters for process implementation. Among other things, digital twins use can significantly save resources on hypothesis testing - it is enough to model the digital double in detail, without making any changes to the actual production and real technological processes.

\section{Methods}

\subsection{Architectural approach}

Description and possibilities (methodology) of applying the architectural approach.

Under the architectural approach in this work is understood a certain set of principles and methods that can ensure a high level of organization of business processes and IT infrastructure at the enterprise, as well as achieving the intended strategic and operational goals in the selected activities based on modern information systems [1].

It is not always possible to limit oneself only to the modernization of existing systems; it is often advisable to reengineer business processes, introduce conceptually new systems, applications and services, which may

\footnotetext{
${ }^{*}$ Corresponding author: aksenovaax@mail.ru
} 
entail the need to ensure correct integration between systems.

The architectural approach is based on the principles of enterprise architecture, which includes 3 layers [2]:

- Business layer;

- IT layer (applications);

- Technological layer (technological and IT infrastructure).

The implementation of architectural solutions allows you to achieve the business goals of the company. At the same time, the enterprise architecture should be flexible enough to adapt in a timely manner to changing external and internal conditions. In addition, the most important quality of an effective enterprise architecture is the ability to maintain the integrity of the entire system when changing its individual parts.

The architectural approach helps to implement information products and information systems with maximum efficiency and effectiveness, since it allows you to build exactly the level of communication between business and IT, at which it is possible to freely implement measures for modeling, implementation and integration of balanced architectural solutions. Today it becomes very difficult to implement various (even small within the company) IT solutions at large enterprises, since they must correctly and effectively interact with existing systems. It is necessary to design the organization and data structure that these products will process and which they will provide at the output. In addition, it is necessary to think over exactly how and in which systems the output data will continue to come, how they will be aggregated and analyzed as part of integration into existing systems. It is these tasks that are successfully implemented using the architectural approach.

In the conditions of constant complication of products and technological processes, modern large industrial enterprises are forced to introduce the most advanced technologies that are not easy to develop and put into practice - they must be embedded in the processes of current systems, which, in turn, must have sufficient flexibility to ensure maximum efficiency from the introduction of new processes [3].

In particular, high-tech companies in the industrial sector are successfully using digital twins - highly detailed models of real objects and their properties for effective hypothesis testing, predicting the values of necessary indicators under given conditions, solving problems of finding optimal configurations and other problems of various spectra. However, it is not enough to develop a digital double; an urgent problem is its integration into existing systems at the enterprise. Often this requires reengineering business processes and changing the organizational structure of the enterprise.

It is for these reasons, in the process of creating a process process data center, it is necessary first of all [4]:

- To formulate goals, the achievement of which will be realized through the CS;

- Creating a model of existing business processes within the framework of which the data center will be used;
- Determine the purpose of the use of the obtained output data of the data center and enter them into existing business processes;

- Solve the problem of coordination of users of input and output data, revise the business functions and organizational structure of the units of the technological unit.

\subsection{Simulation of real technological processes using data, incl. - in data architecture and systems and application architecture}

Digital twins are created, first of all, to ensure the saving of time, labor and financial resources of the enterprise. For example, in German enterprises over the past 7 years, digital twins have reduced the number of tests in the field by 50 times [5]. In this case, it was digital twins that underwent testing, which allowed to reduce the number of people involved in the tests by more than an order of magnitude. In addition, the introduction of digital twins is quite strongly reflected not only in the number of personnel, but also in its structure. So, the ratio of specialists in design engineers and calculating analysts in favor of the latter is changing dramatically. If earlier for every 5 designers there was one counter, now now for each designer there are already two counters.

A digital double is capable of generating in real time hundreds of thousands of different scenarios related to the prototype depending on the desired task. And at the same time, you do not need to create a material copy and test it in real conditions.

Today, digital twins are becoming more and more complicated: along with digital twins , for example, products or individual equipment, digital twins of entire sectors or production sites arise, covering the entire internal ecosystem of production processes in individual areas. Such complex systems require a certain approach to their development and implementation in existing enterprise processes. Since often, due to the lack of competent specialists in the field of digital twins at the enterprise, the development is outsourced to a few companies and research centers specializing in these tasks, many problems may arise that need to be addressed:

- It is not always clear how to integrate new digital twin applications and existing systems;

- The task of identifying new roles in the organizational structure: it is not clear who should be the responsible person, as well as the person making decisions in the field of interaction with the digital double;

- Improving the efficiency of using the output of a digital double, solving the problem of finding the optimal solution, etc.

\subsection{Building an architectural process control model incorporating a digital double}

Architectural model on the example of CD of compression and low-temperature separation of associated petroleum gas and natural gas 
When realizing the task of creating a digital double of the technological process, it is advisable to be based on the principles of the architectural approach, which implies the comprehensive integration of data centers into the enterprise's IT architecture.

Providing effective process control based on the collection and analysis of process data implies [6]:

- formation of requirements for the structure of input and output data;

- formation of requirements and regulation of methods for their collection, processing, analysis;

- formation of managerial influences and methods of predictive or reactive response;

- selection of the most effective parameters of the iterative process under different experimental conditions of the external and internal environment.

The digital double operation scheme involves the collection of data from equipment sensors that come through information systems at the level of Automated Process Control Systems (APCS). Further, these data are processed at the level of Production Process Control Systems (MES) to carry out the following process control functions:

- Analysis of output data in real time;

- Selection of optimal parameters and equipment configurations;

- Prediction of the necessary configurations of a digital double, output parameters;

- Making management decisions based on analysis and forecast data.

After processing the data at the MES level, the information enters the systems of the Company's Resource Management (ERP) level, where the data is analyzed to make operational decisions on managing financial, human and other resources of the enterprise), then the aggregated and processed data is sent to Business Intelligence IS (BI ), the analysis of which is already being considered at the highest level of the company's top management for making strategic decisions on business management.

As a result of the analysis of the data obtained using the digital double, the optimal configuration of the operating modes of the individual elements of the station and installation is automatically set for real equipment.

As an example of the implementation of a digital double implemented at a real enterprise in the oil and gas industry, this article provides a program simulating gas infrastructure developed at the Department of Compressor, Vacuum and Refrigeration Engineering of Peter the Great St. Petersburg Polytechnic University (hereinafter referred to as KViHT Department) [7]. The software product was developed on the basis of a schematic diagram that includes a technological diagram of piping, the main and auxiliary equipment of two jointly (sequentially) working gas infrastructure facilities: a high-pressure gas compressor station and a low-temperature gas treatment unit. A significant array of program elements, in addition to compressor, turboexpander, heat transfer, drainage and separation equipment, is piping and the operation of these elements of the software product is based on a mathematical model of losses in pipelines [8]. On the branched and looped sections of the network, a specially developed mathematical model of the distribution of costs on the branched and ring sections of the network is applied. Separate program modules are responsible for the operation of dynamic and volume compressors, based on the developed features of the characteristics of these types of compressors. The characteristics of the compressor equipment were obtained during field experiments at the research object and using mathematical modeling in the proprietary software products of the Department of QuiHT and using CFD modeling in the Ansys CFX [21, 22, 23, 24] software package. Modeling of separation and heat exchange equipment is represented by mathematical models of auxiliary equipment. In the program, in addition to the main modules, there are additional modules responsible for opening and closing various shut-off and control valves. These modules allow you to adjust the operating modes of the station and determine the most effective percent opening valves and operating modes of the equipment.

\subsection{Digital twin of high-tech compression and low-temperature separation processes}

\subsubsection{Requirements description for digital twin}

There are three main requirements for CD:

1. to the input and output data;

2. to the structure;

3. to software (according to GOST 34.602).

It is necessary to say separately about the first requirement. The schematic diagram of simulation objects is converted to SVG format and each element of such a circuit is fully interactive and has its own properties. Some elements of the system are intended only for visual data output - these are sections of pipelines or auxiliary equipment that do not have optional regulation functions. Other elements of the system are designed both for output and for data input these are the main (compressor), auxiliary equipment and control valves. An example of a digital twin interface is shown in Figure 1.

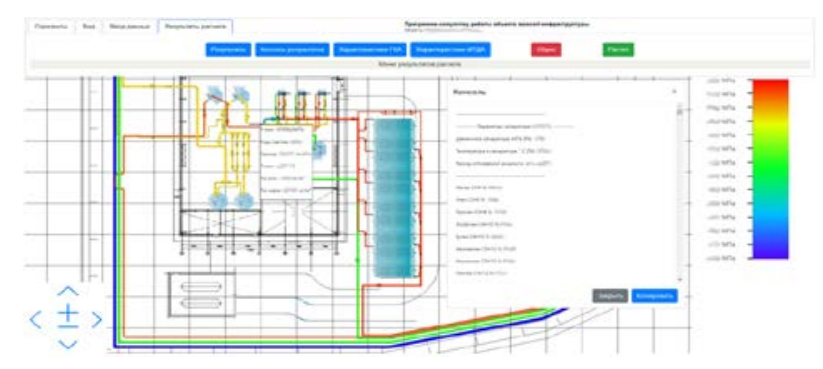

Fig.1. Digital twin interface [7].

\subsubsection{Data input}

- Input amount is presented in several categories:

- Environmental parameters;

- One or more working environment inputs parameters; 
- Mechanical parameters the simulated network objects regulation;

- Network design parameters and network elements (to optimize design decisions);

- Output network parameters.

The first four categories of data entry are implemented in the gas infrastructure simulator program as a direct simulation task (from input parameters to obtain output parameters). The fifth category of regulation with input parameters of the output network is the most complex and as close as possible digital double of a real gas infrastructure object (inverse problem).

\subsubsection{Data output}

- Output amount is presented in several categories:

- Thermo-gas-dynamic medium parameters;

- Network design parameters and network elements;

- Energy consumption calculated parameters;

- Efficiency design parameters and equipment reliable operation.

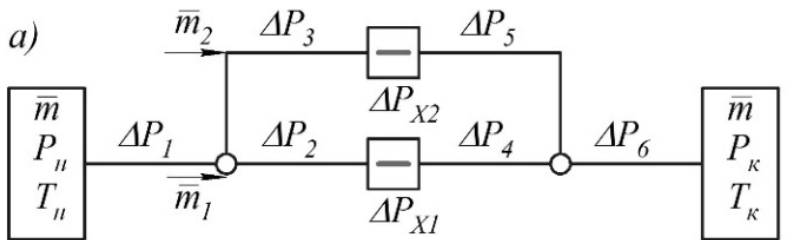

$$
P_{\kappa}=P_{H}-\Delta P_{1}-\Delta P_{6}-\Delta P_{\Sigma} \text {, }
$$

where $\Delta P_{\Sigma}=f\left(\bar{m}_{1}\right)=\left(\Delta P_{2}+\Delta P_{4}+\Delta P_{X I}\right)$

$\Delta P_{\Sigma}=f\left(\bar{m}_{2}\right)=\left(\Delta P_{3}+\Delta P_{5}+\Delta P_{X 2}\right)$

$\bar{m}=\bar{m}_{1}+\bar{m}_{2}$

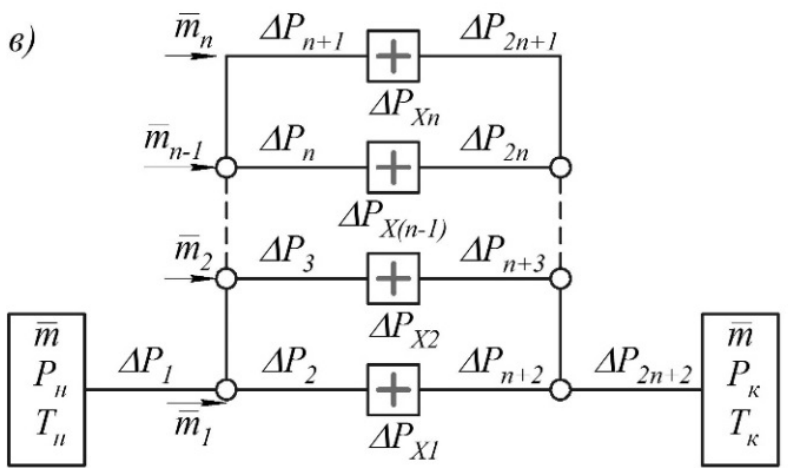

$$
P_{\kappa}=P_{H}-\Delta P_{I}-\Delta P_{2 n+2}+\Delta P_{\Sigma},
$$$$
\text { where } \Delta P_{\Sigma}=f\left(\bar{m}_{1}\right)=\left(\Delta P_{2}+\Delta P_{n \mid 2}-\Delta P_{X 1}\right)
$$$$
\Delta P_{\Sigma}=f\left(\bar{m}_{2}\right)=\left(\Delta P_{3}+\Delta P_{n ! 3}-\Delta P_{X 2}\right)
$$$$
\Delta P_{\Sigma}=f\left(\bar{m}_{n-l}\right)=\left(\Delta P_{n}+\Delta P_{2 n}-\Delta P_{X(n-l)}\right)
$$$$
\Delta P_{\Sigma}=f\left(\bar{m}_{n}\right)=\left(\Delta P_{n+1}+\Delta P_{2 n+1}-\Delta P_{X n}\right)
$$$$
\bar{m}=\bar{m}_{1}+\bar{m}_{2}+\ldots+\bar{m}_{n-1}+\bar{m}_{n}
$$

At a real facility, it is not possible to analyze the thermo-gas-dynamic parameters of the medium over the length of the entire gas pipeline network of the facility. Only at the control points (entry and exit) of the main and auxiliary equipment, as well as at the exit from separate sections of the station, it is possible to carry out measurements of gas-dynamic parameters and analysis of the environment to monitor the operation of the station. Thus, the digital double should produce a separate data output at all the reference points of the system (measurement areas of the natural object of study). This approach simplifies the possibility of validating a digital double at the object of study.

\subsubsection{Additional interface features}

Implemented features:

- output of the main thermodynamic parameters in the color palette in a given range of values (visualization of thermodynamic parameters);

- a graphical view of the characteristics of the

б)

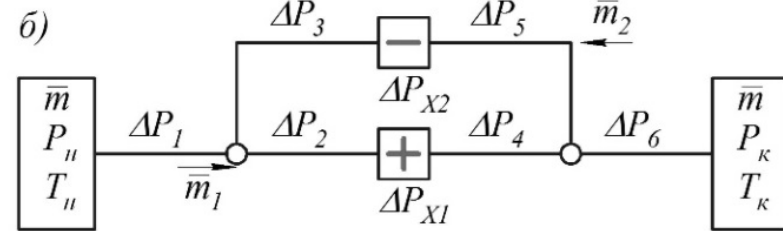

$$
P_{\kappa}=P_{H}-\Delta P_{I}-\Delta P_{6}+\Delta P_{\Sigma} \text {, }
$$$$
\text { where } \Delta P_{\Sigma}=f\left(\bar{m}_{1}\right)=\left(\Delta P_{2}+\Delta P_{4}-\Delta P_{X 1}\right)
$$$$
\Delta P_{\Sigma}=f\left(\bar{m}_{2}\right)=-\left(\Delta P_{3}+\Delta P_{5}+\Delta P_{X 2}\right)
$$$$
\bar{m}=\bar{m}_{l}-\bar{m}_{2}
$$

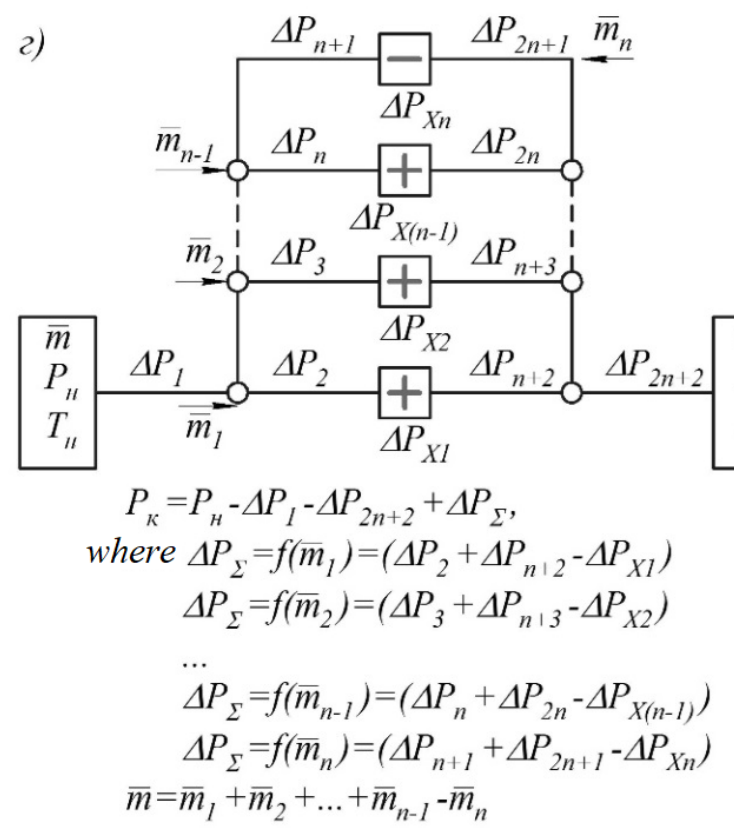

Fig. 2. Parallel flow distribution block diagram: a) parallel distribution with adjustable resistance (valve - $\square$ ); b) parallel distribution with adjustable resistance (bypass - - ) and pressure increase (compressor - + ); c) n-sections parallel distribution with increasing pressure (compressor - + ); d) n-sections parallel distribution with increasing pressure (compressor $-\square$ ) and with one adjustable resistance (bypass $-\square$ ). 
equipment (equipment performance);

- input of system control input parameters from a file;

Saving the changed parameters of the system regulation in a file with the possibility of further loading.

Opportunities for digitalization:

- saving all simulation results to a database;

- analysis and optimization of the results in a database of optimization factors (economic efficiency, resource, etc.);

- the introduction of optimal results in the modeling process with the inclusion of optimization factors.

\section{Results}

Key work models are based on the determination of pressure and temperature losses in the sections of the

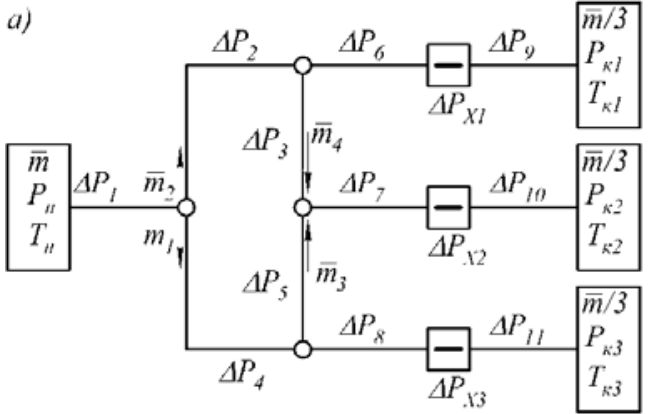

$P_{\kappa l}=P_{n}-\Delta P_{1}-\Delta P_{2}-\Delta P_{27}$.

where $\Delta P_{51}=f(m / 3)=\Delta P_{6}+\Delta P_{9}+\Delta P_{X 7}$

$$
\Delta P_{2}=f\left(m_{2}\right)
$$

$P_{k 3}=P_{u}-\Delta P_{1}-\Delta P_{4}-\Delta P_{\Sigma 3}$

where $\Delta P_{23}=f(\bar{m} / 3)=\Delta P_{g}+\Delta P_{11}+\Delta P_{X 3}$ $\Delta P_{4}=f\left(\bar{m}_{1}\right)$

$P_{k 2}=P_{n}-\Delta P_{1}-\Delta P_{x}-\Delta P_{23}$,

where $\Delta P_{52}=f(m / 3)=\Delta P_{7}+\Delta P_{10}+\Delta P_{X 2}$ $\Delta P_{x}=\Delta P_{2}+\Delta P_{3}=\Delta P_{4}+\Delta P_{5}$ $\Delta P_{3}=f\left(m_{4}\right) ; \Delta P_{5}=f\left(m_{3}\right)$

$m=m_{1}+m_{2} ; m_{3}+m_{4}=m / 3$

$; \bar{m}_{1}-\bar{m}_{3}=\bar{m} / 3$

; $m_{2}-m_{4}=m / 3$ б)

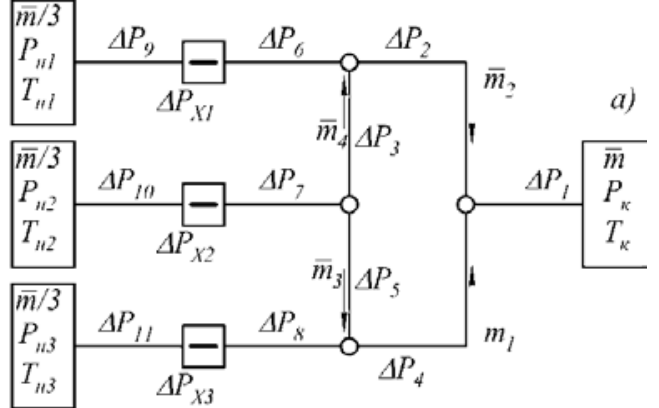

$P_{k}=P_{u l}+\Delta P_{2 l}+\Delta P_{2}+\Delta P_{l}$,

where $\Delta P_{\Sigma I}=f(m / 3)=\Delta P_{6}+\Delta P_{9}+\Delta P_{X I}$

$$
\Delta P_{2}=f\left(m_{2}\right)
$$

$P_{\kappa}=P_{n 3}+\Delta P_{53}+\Delta P_{4}+\Delta P_{l}$,

where $\Delta P_{23}=f(\bar{m} / 3)=\Delta P_{8}+\Delta P_{11}+\Delta P_{X 3}$

$$
\Delta P_{4}=f\left(\bar{m}_{l}\right)
$$

$P_{k}=P_{u,} \Delta P_{w}+\Delta P_{x}+\Delta P_{l}$,

where $\Delta P_{\Sigma 2}=f(m / 3)=\Delta P_{7}+\Delta P_{10}+\Delta P_{X 2}$

$$
\Delta P_{x}=\Delta P_{2}+\Delta P_{3}=\Delta P_{4}+\Delta P_{5}
$$$$
\Delta P_{3}=f\left(m_{4}\right) ; \Delta P_{5}=f\left(m_{3}\right)
$$

$m=m_{1}+m_{2} ; m_{3}+m_{4}=m / 3$

$$
; m_{1}-m_{3}=m / 3
$$

; $m_{2},-m_{4}=m / 3$

$$
\begin{aligned}
& \text { 6) } \\
& P_{k}=P_{n}-\Delta P_{l}-\Delta P_{2}-\Delta P_{\Sigma l}-\Delta P_{15}-\Delta P_{l 6} \text {, где } \Delta P_{\Sigma l}=f\left(\bar{m}_{x l}\right)=\left(\Delta P_{6}+\Delta P_{95}-\Delta P_{X l}\right) \\
& P_{k}=P_{l i}-\Delta P_{l}-\Delta P_{4}-\Delta P_{23}-\Delta P_{l 4}-\Delta P_{l 6} \text {, zde } \Delta P_{23}=f\left(m_{x 3}\right)=\left(\Delta P_{8}+\Delta P_{1 /}-\Delta P_{13}\right) \\
& P_{K}=P_{11}-\Delta P_{1}-\Delta P_{2}-\Delta P_{3}-\Delta P_{22}-\Delta P_{13}-\Delta P_{15}-\Delta P_{16} \text {, } \\
& P_{n}=P_{n}-\Delta P_{1}-\Delta P_{4}-\Delta P_{5}-\Delta P_{52}-\Delta P_{12}-\Delta P_{14}-\Delta P_{16} \text {, } \\
& \text { where } \Delta P_{E 2}=f\left(\bar{m}_{x 3}\right)=\left(\Delta P_{s}+\Delta P_{11}-\Delta P_{13}\right) \\
& \Delta P_{2}=f\left(\bar{m}_{2}\right) ; \Delta P_{3}=f\left(\bar{m}_{4}\right) ; \Delta P_{4}=f\left(\bar{m}_{1}\right) ; \Delta P_{5}=f\left(\bar{m}_{3}\right) \\
& \Delta P_{12}=f\left(\bar{m}_{6}\right) ; \Delta P_{13}=f\left(\bar{m}_{5}\right) ; \Delta P_{14}=f\left(\bar{m}_{7}\right) ; \Delta P_{15}=f\left(\bar{m}_{8}\right) \\
& \bar{m}=\bar{m}_{1}+\bar{m}_{2}=\bar{m}_{g}+\bar{m}_{7}=\bar{m}_{x l}+\bar{m}_{x 2}+\bar{m}_{x 3} \\
& \bar{m}_{x l}=\bar{m}_{2}-\bar{m}_{4}=\bar{m}_{g}-\bar{m}_{5} \\
& m_{x 2}=m_{3}+m_{4}=m_{6}+m_{5} \\
& \bar{m}_{x 3}=\bar{m}_{l}-\bar{m}_{3}=\bar{m}_{7}-\bar{m}_{6}
\end{aligned}
$$

Fig. 3. Ring flow distribution block diagram:a) input ring collector with adjustable resistance (valve - $\square$ ); b) output ring collector with adjustable resistance (valve - - ); c) connection diagram through 3 sections ring collectors with increasing pressure (compressor $-\square$ )with one adjustable resistance (bypass $-\square$ ). 
simulated network (first and second models) and the model for determining the thermodynamic parameters of the working medium (third model). The fourth model of the network (model of the expendable component) is used to determine the parameters of the parallel distribution of several flows of the working environment. Namely, the branching, merging and regulation of the flow component of the flow in areas of parallel and sequential operation. The remaining models are designed for the individual operation of complex network elements and are based on a variety of physical and mathematical laws and studies [9, 10,11, 12, etc.].

\subsection{Key work models}

Pressure losses are determined by the Darcy-Weischbach equation - an empirical formula that determines the pressure loss or pressure loss during a developed turbulent flow of incompressible fluid at hydraulic resistance (proposed by Julius Weisbach in 1855).

The Weisbach formula, which determines the pressure loss on the hydraulic resistances, has the form:

$$
\Delta P=\zeta \cdot \frac{\rho c^{2}}{2},
$$

where $\zeta$ is the coefficient of hydraulic resistance, $\rho$ is the density of the working medium (gas), and c is the flow rate of the working medium (gas).

The temperature loss from the environment is determined according to the conditions of heat and mass transfer between the turbulent flow of the working medium and the external laminar air flow through the wall of the metal pipe and the heat-insulating layer of different types and thicknesses.

The calculations of the thermodynamic properties of the working medium are based on the equations of thermodynamic calculation of the BIE (equation of state of Benedict, Webb, Rubin) and its modifications [13]. Modifications are based on the approach of using the equation of state [14]:

- Process definition and its properties from input and output parameters;

- Output determination parameters by isoenthalpic process;

- Output determination parameters by polytropic process.

\subsection{The model of the expendable component of the flow of the working environment}

The work of mathematical models of the distribution of the expendable component (flow) of the working medium flow over the branched sections of the network $[15,16,17,18,19,20]$ is presented in the form of a multi-stage, multi-iteration calculation using a software modification of the gradient descent method. The stopping condition is the equality of the pressure loss in all separate parallel directions, and the magnitude of the first approximation is the expenditure component in each direction and the invariance of the flow rate in all directions. The block diagram of the models of distribution and flow control are presented in Figures 2 and 3.

The obtained regulatory models require the correct ratio regulatory influence and the resistance coefficient $(\zeta)$. All control elements have a programmed function $\zeta=\mathrm{f}(\mathrm{x} 1 ; \mathrm{x} 2 ; \ldots ; \mathrm{xn})$, where $(\mathrm{x})$ is the control value and such values for one control element may have one or more. In the case of further interface capabilities implementation for digitalization, the regulation values for the digitalization object will be selected from the optimization criteria or criterion. It is possible to direct this value as an analog signal from a virtual simulator to an existing object.

\section{Conclusions}

The digital double as an integral part of the production process of the enterprise should be developed and implemented as a system and integrated project. In turn, projects of such high complexity as a digital double should be implemented based on the methods of the architectural approach, since it is difficult to integrate new processes in high-level and multidimensional organizational structures of large industrial enterprises. Using the architectural approach, it is possible to achieve high-quality integration of the created digital double with existing information systems, so that its effective application in the enterprise becomes available.

The work justified the feasibility of applying the architectural approach in the development and implementation of digital twins, and also developed recommendations regarding the location of the digital double in the architecture of information systems of a manufacturing enterprise.

\section{References}

1. Ilin, I., Levina, A., \& Iliashenko, O. (2018). Enterprise architecture analysis for energy efficiency of Saint-Petersburg underground. Advances in Intelligent Systems and Computing, 692, 1214-1223. doi:10.1007/978-3-319-709871_130

2. lin, I., Levina, A., Abran, A., Iliashenko, O. (2017). Measurement of enterprise architecture (EA) from an IT perspective: Research gaps and measurement avenues. Paper presented at the ACM International Conference Proceeding Series, Part F131936, 232243. doi:10.1145/3143434.3143457

3. Ilin, I., Levina, A., \& Iliashenko, O. (2017). Enterprise architecture approach to mining companies engineering. Paper presented at the MATEC Web of Conferences, 106, Article number 08066. doi:10.1051/matecconf/201710608066

4. Ilin, I. V., Iliashenko, O. Y., \& Borremans, A. D. (2017). Analysis of cloud-based tools adaptation possibility within the software development projects. Paper presented at the Proceedings of the 
30th International Business Information Management Association Conference, IBIMA 2017 - Vision 2020: Sustainable Economic Development, Innovation Management, and Global Growth, 2017January, 2729-2739.

5. Ilin, I., Levina, A., Lepekhin, A., Kalyazina, S. Business Requirements to the IT Architecture: A Case of a Healthcare Organization (2019) Advances in Intelligent Systems and Computing, 983, pp. 287294.

6. Levina, A.I., Borremans, A.D., Burmistrov, A.N. Features of enterprise architecture designing of infrastructure-intensive companies (2018) Proceedings of the 31st International Business Information Management Association Conference, IBIMA 2018: Innovation Management and Education Excellence through Vision 2020, pp. 4643-4651.

7. Biktimirov A.A., Aksenov A.A., Kozhukhov Yu.V. Creation of a "digital twin" of gas infrastructure facilities at the Vankor field. Proceedings of the 21st annual international industrial symposium "Compressors and compressor equipment" named after K.P. Selezneva. 2019.163 p.

8. Idelchik I. Ye. Handbook of hydraulic resistance / Ed. M.O. Shteinberg. - 3rd ed., Rev. and add. - M .; Engineering, $1992.672 \mathrm{~s}$.

9. A.A. Lebedev, Yu.V. Covers. The development of a centrifugal compressor protection system against surge in an indicative way using computational fluid dynamics and analysis of pressure pulsation signals. Gas industry. No. 6 (753) / 2017. Ed. CJSC Camelot Publishing. S. 60 - 66.

10. A.M. Yablokov, Yu.V. Kozhukhov, A.A. Lebedev. Investigation of the flow in a low-flow stage of a centrifugal compressor using computational gas dynamics methods. Scientific and technical statements of SPbSPU. Vol. 4 (231) / 2015. Ed. SPbSPU. S. 59 - 69.

11. E.G. Kozin, Yu.V. Covers. Technological audit of the pneumatic system as a stage in the process of its reconstruction. Scientific and technical statements of SPbSPU. Vol. 2 (243) / 2016. Ed. SPbSPU. S. 34 44.

12. Denisenko VV, Mironov Yu.R., Malev K.G., Kozhukhov Yu.V., Danilishin A.M., Kartashov S.V., Neverov V.V. The task of validating the gasdynamic characteristics of a multistage centrifugal compressor of a booster compressor station of natural gas. / Proceedings of the international symposium "Compressors and compressor equipment" named. K.P. Selezneva. Ministry of Education; SPbPU. 2018. Publisher: Polytech Press.

13. Aksenov A.A., Sokolov M.I., Chernyshev M.S., Nazarenko N.A. Analysis and modernization of the program for thermodynamic calculation of real gas based on modified Benedict-Webb-Rubin equations. SPbPU Science Week: proceedings of a scientific conference with international participation. Institute of Energy and Transport Systems. Part 1. - SPb .: Publishing house of the Polytechnic. University, 2017. 248s

14. Aksenov A., Kozhukhov Y., Sokolov M., Simonov A. Analysis and modernization of real gas thermodynamic calculation for turbocompressors and detander units. (2018) MATEC Web of Conferences, 245, article No. 09005. DOI: 10.1051 / matecconf / 201824509005

15. Kozhukhov Yu.V., Lebedev A.A., Sadovsky N.I., Kartashov S.V. Stations and pneumatic networks: compressor, drive and auxiliary equipment: educational / Yu.V. Kozhukhov [et al.]. - SPb .: Publishing house of the Polytechnic. University, 2018.98 s.

16. Bleicher I.G., Liseev V.P. “Compressor stations” Moscow: Mashgiz, 1959.

17. Aksenov A.A., Sokolov M.I., Chernyshev M.S., Nazarenko N.A. Analysis and modernization of the program for thermodynamic calculation of real gas based on modified Benedict-Webb-Rubin equations. SPbPU Science Week: proceedings of a scientific conference with international participation. Institute of Energy and Transport Systems. - SPb .: Publishing house of the Polytechnic University, 2017 . 248c.

18. H. Krain, "A study on centrifugal impeller and diffuser flow,” Journal of Engineering for Power, vol. 103, no. 4, pp. 688-697, 1981 DOI: 10.1115/1.3230791.

19. Z. Guzović, M. Baburić, and D. Matijašević, "Comparison of flow characteristics of centrifugal compressors by numerical modelling of flow," Journal of Mechanical Engineering, vol. 51, no. 7-8, pp. 509-518, 2005.

20. B. E. Launder and D. B. Spalding, "The numerical computation of turbulent flows," Computer Methods in Applied Mechanics and Engineering, vol. 3, no. 2, pp. 269-289, 1974. DOI: 10.1016/00457825(74)90029-2

21. Ivanov, E.A., Zharkovsky, A.A., Borshchev, I.O., Svoboda, D.G. Technique for axial pump characteristics predicting in CFD package Openfoam. AIP Conference Proceedings v.2141,\#030063. (2019). DOI: 10.1063/1.5122113.

22. Zhurkin, N., Donskoj, A., Zharkovskij, A. Numeric modeling and estimating the performance characteristics of a pneumatic driven high pressure pump. (2018) MATEC Web of Conferences, 245, \# 09014. DOI: 10.1051/matecconf/201824509014.

23. Zharkovskii, A.A., Pospelov, A.Y. Use of 3D Methods for Flow Analysis, Prediction of Characteristics, and Optimization of the Shape of Settings of Hydraulic Turbines. (2015) Power Technology and Engineering, 49 (1), pp. 27-32.

24. Pospelov, A.Y., Zharkovskii, A.A. Effect of the Parameters of a Computational Model on the Prediction of Hydraulic Turbine Characteristics. 
(2015) Power Technology and Engineering, 49 (3), pp. 159-164. 